\title{
INTERVENÇÕES DE ENFERMAGEM PARA PREVENÇÃO DE QUEDAS EM IDOSOS NA ATENÇÃO PRIMÁRIA: REVISÃO INTEGRATIVA
}

\section{NURSING INTERVENTIONS FOR THE PREVENTION OF FALLS IN THE ELDERLY IN PRIMARY CARE: INTEGRATIVE REVIEW}

\section{Paulo Henrique Fernandes dos Santos ${ }^{1} *$ Denise Galvão Sampaio ${ }^{2} *$ Marina Morato Stival $^{3}$ Luciano Ramos de Lima ${ }^{4}$ *Walterlânia Silva Santos ${ }^{5} *$ Silvana Schwerz Funghetto $^{6}$}

\begin{abstract}
RESUMO
Objetivo: identificar as intervenções de enfermagem propostas para prevenir quedas em idosos no contexto da atenção primária. Métodos: revisão integrativa da literatura cujos estudos foram identificados nas bases de dados PubMed, CINAHL e LILACS em abril de 2020. Resultados: foram selecionados quinze artigos, dos quais emergiram duas temáticas principais: intervenções de enfermagem relacionadas à avaliação e gestão do risco de quedas em idosos na atenção primária; intervenções de enfermagem em programas multiprofissionais para prevenção de quedas em idosos na atenção primária. Considerações finais: as intervenções de enfermagem para prevenção das quedas no público estudado consistem em avaliar o risco de quedas; orientar idosos/cuidadores quanto a promoção da segurança no domicílio e mudanças comportamentais; promover educação permanente para a equipe de saúde sobre a temática; conduzir e/ou participar de programas multiprofissionais com a finalidade de prevenir as quedas. Estes achados podem contribuir para o planejamento das ações dos profissionais que se deparam cotidianamente com o desafio de prevenir as quedas em idosos na atenção primária.
\end{abstract}

Palavras-chave: Acidentes por Quedas; Idoso; Enfermagem; Cuidados de Enfermagem; Atenção Primária à Saúde.

\begin{abstract}
Keywords: Accidental Falls; Aged; Nursing; Nursing Care; Primary Health Care.

\footnotetext{
${ }^{1}$ Universidade de Brasília, Brasília, Brasil. ORCID: https://orcid.org/0000-0003-4533-0129

${ }^{2}$ Universidade de Brasília, Brasília, Brasil. ORCID: https://orcid.org/0000-0002-2005-3834

${ }^{3}$ Universidade de Brasília, Brasília, Brasil. ORCID: https://orcid.org/0000-0001-6830-4914

${ }^{4}$ Universidade de Brasília, Brasília, Brasil. ORCID: https://orcid.org/0000-0002-2709-6335

${ }^{5}$ Universidade de Brasília, Brasília, Brasil. ORCID: https://orcid.org/0000-0001-6266-8901

${ }^{6}$ Universidade de Brasília, Brasília, Brasil. ORCID: https://orcid.org/0000-0002-9332-9029
}

Objective: to identify nursing interventions proposed to prevent falls in the elderly in the context of primary care. Methods: integrative review of the literature whose studies were identified in the PubMed, CINAHL and LILACS databases in April 2020. Results: fifteen articles were selected, from which two main themes emerged: nursing interventions related to the assessment and risk management of falls in the elderly in primary care; nursing interventions in multidisciplinary programs to prevent falls in the elderly in primary care. Final considerations: nursing interventions to prevent falls in the studied public consist of assessing the risk of falls; guide elderly/caregivers regarding the promotion of home safety and behavioral changes; promote continuing education for the health team on the theme; conduct and/or participate in multi-professional programmes with the aim of preventing falls. These findings can contribute to the planning of the actions of professionals who face the challenge of preventing falls in the elderly in primary care on a daily basis. 


\section{INTRODUÇÃO}

As quedas consistem em eventos nos quais o indivíduo cai no solo ou em níveis inferiores, de modo inadvertido, exceto as mudanças intencionais de posição para apoiar-se em móveis, paredes ou outros objetos. São consideradas um importante problema de saúde pública, uma questão prioritária na área da saúde em âmbito mundial e estão relacionadas principalmente à população idosa, tendo em vista as alterações morfológicas e fisiológicas inerentes ao processo de envelhecimento que potencializam a vulnerabilidade a esses eventos $^{(1)}$.

Segundo a Organização Mundial de Saúde (OMS), as quedas são a segunda causa de morte acidental ou não intencional em todo o mundo, e os idosos com 65 anos ou mais são os que mais sofrem com as quedas fatais $^{(2)}$. Os dados referentes aos episódios de quedas nesta população são alarmantes e indicam que entre 28 e $35 \%$ das pessoas com idade $\geq 65$ anos vivenciam, ao menos, um episódio de queda ao ano, e naquelas com idade $\geq 70$ anos, a estimativa aumenta para 32 a $42 \%$. Estima-se que cerca de $5 \%$ das quedas em idosos na comunidade ocasionam fraturas, e de 5 a $10 \%$ ocasionam lesões de tecidos moles e cabeça ${ }^{(1)}$.

Diversos fatores contribuem para a ocorrência das quedas. Estes fatores podem ser classificados em intrínsecos, relacionados às características inerentes ao indivíduo como idade avançada, doenças e medo de cair; e em extrínsecos, que dizem respeito à interação com o meio ambiente, como pisos escorregadios, iluminação insuficiente, entre outros. Ressalta-se que os fatores extrínsecos são considerados modificáveis, logo, são alvo das ações preventivas ${ }^{(3-4)}$.

Considerando que as quedas são eventos evitáveis, torna-se essencial a adoção de ações preventivas, por exemplo, a identificação dos fatores de risco $^{(5)}$. Neste contexto, destaca-se o trabalho dos enfermeiros na prevenção das quedas em idosos, tendo em vista sua notória atuação na atenção primária. $\mathrm{O}$ enfermeiro apresenta competência para realizar julgamento clínico e estabelecer Diagnósticos de Enfermagem (DE) precisos - por exemplo, os DE da CIPE Risco de Queda e Risco de Lesão por Queda $^{(6)}$, ou o DE da NANDA-I Risco de Quedas $^{(7)}$, o que favorece o estabelecimento de intervenções com objetivo de prevenir as quedas e suas consequências ${ }^{(8)}$.

Cabe ressaltar que a OMS considera a prevenção das quedas como uma das metas internacionais de segurança do paciente. Além disso, o Programa Nacional de Segurança do Paciente, criado em 2013 pelo Ministério da Saúde, Anvisa e Fiocruz, instituiu o Protocolo de Prevenção de Quedas com a finalidade de reduzir a ocorrência desses eventos e dos seus 
$\operatorname{danos}^{(9)}$, entretanto, as medidas preventivas propostas são voltadas para o contexto hospitalar, havendo uma lacuna no que diz respeito às ações que se adequam às especificidades dos idosos atendidos na atenção primária.

Esta mesma lacuna é observada na literatura de enfermagem, na qual são encontrados estudos destinados à abordagem das quedas (contemplando diagnósticos, resultados e intervenções de enfermagem) em idosos institucionalizados ${ }^{(10)} \quad \mathrm{e}$ $\operatorname{hospitalizados}^{(8-11)}$, porém, ainda são escassos os trabalhos realizados com idosos da comunidade e que sintetizam o conhecimento sobre as intervenções de enfermagem para prevenção das quedas.

Diante do exposto o objetivo desta pesquisa foi identificar as intervenções de enfermagem propostas para prevenção de quedas em idosos no contexto da atenção primária à saúde disponíveis na literatura.

\section{MÉTODOS}

Trata-se de uma revisão integrativa da literatura, um tipo de investigação utilizada na Prática Baseada em Evidências, que inclui a busca, análise crítica e a síntese das evidências científicas disponíveis sobre determinado tema ou questão, visando a incorporação de novos conhecimentos na prática clínica ${ }^{(12)}$.
A presente revisão foi desenvolvida em seis etapas: identificação do tema e definição da pergunta de pesquisa; escolha dos critérios de inclusão e exclusão dos estudos; definição das informações que serão extraídas dos estudos; avaliação dos estudos incluídos na amostra; interpretação dos resultados e apresentação da revisão ${ }^{(12)}$.

A pergunta de pesquisa foi delimitada com auxílio da estratégia PCC, dessa forma, considerou-se $\mathrm{P}$ (paciente): idosos; $\mathrm{C}$ (conceito): intervenções de enfermagem para prevenir as quedas; C (contexto): atenção primária à saúde. Logo, a pergunta de pesquisa foi "Quais as intervenções de enfermagem propostas para prevenir as quedas em idosos no contexto da atenção primária à saúde?" (13).

Em seguida, foram definidos os critérios de elegibilidade dos estudos. Os critérios de inclusão consistiram em: estudos desenvolvidos com população idosa (idade $\geq$ 60 anos); que abordassem intervenções de enfermagem para prevenir quedas; realizado no contexto da atenção primária; publicados nos idiomas português, espanhol ou inglês; disponibilizados na íntegra. Foram excluídos estudos com outras populações (adultos), no contexto de institucionalização e/ou hospitalização, além de revisões, relatos de experiência, opiniões de editores, publicações de anais de congressos e editoriais. 
As buscas foram realizadas em abril de 2020 nas bases de dados PubMed, CINAHL e LILACS com os descritores DeCS e MeSH: aged, accidental falls, primary health care, nursing, nursing care. Estes descritores foram combinados empregando os operadores boleanos $A N D$ e $O R$ para compor as estratégias de busca, de acordo com as especificidades de cada base de dados. Para a LILACS foram incluídos os descritores nos idiomas português e espanhol (Quadro 1).

Quadro 1 - Estratégias de busca, de acordo com as bases de dados selecionadas.

\begin{tabular}{|c|c|}
\hline Base de dados & Estratégia de Busca \\
\hline PubMed & $\begin{array}{l}\text { ("aged"[MeSH Terms] OR "aged" [All fields]) AND } \\
\text { ("accidental falls"[MeSH Terms] OR "accidental falls" [All } \\
\text { fields]) AND ("primary health care"[MeSH Terms] OR } \\
\text { "primary health care"[All fields]) AND ("nursing"[MeSH } \\
\text { Terms] OR "nursing”"[All fields]) }\end{array}$ \\
\hline CINAHL & $\begin{array}{l}\text { "aged" AND "accidental falls" AND "primary health care" } \\
\text { AND "nursing" }\end{array}$ \\
\hline LILACS & $\begin{array}{l}\text { ("Aged" OR “Anciano" OR "Idoso") AND ("Accidental Falls" } \\
\text { OR "Accidentes por Caidas" OR "Acidentes por Quedas") } \\
\text { AND ("Primary Health Care" OR "Atención Primaria de } \\
\text { Salud" OR “Atenção Primária à Saúde") AND ("Nursing” OR } \\
\text { "Enfermería" OR "Enfermagem") }\end{array}$ \\
\hline
\end{tabular}

Fonte: Dados da pesquisa, 2020.

Os estudos que resultaram da busca foram exportados para o gerenciador de referência EndNote Web, no qual procedeu-se com a exclusão das duplicatas. Em seguida, foram exportados para a ferramenta Rayyan, onde realizou-se a leitura e seleção dos títulos e resumos de maneira individual $\mathrm{e}$ independente pela revisora 1 (DGS) e revisor 2 (PHFS). Logo depois, os dois revisores iniciaram a leitura e análise dos artigos na íntegra, para selecionar os estudos que iriam compor a amostra final da revisão. Ao final desses procedimentos, as discordâncias foram resolvidas entre os dois revisores.

Após a seleção dos estudos, foi efetuada a coleta dos dados em uma planilha composta pelas seguintes variáveis: autores, ano de publicação, país onde o estudo foi desenvolvido, natureza e tipo de estudo, participantes da pesquisa e, por último, os https://doi.org/10.31011/reaid-2021-v.95-n.34-art.1104 Rev Enferm Atual In Derme v. 95, n. 34, 2021 e-021089 
resultados que permitiam responder a pergunta de pesquisa desta revisão.

Posteriormente, os estudos foram avaliados conforme o nível de evidência: (1) resultados de meta-análise de estudos clínicos controlados e randomizados; (2) evidências obtidas em estudos individuais com delineamento experimental; (3) evidências de estudos quase-experimentais; (4) evidências de estudos descritivos (não-experimentais) ou com abordagem qualitativa; (5) evidências provenientes de relatos de caso ou de experiência; (6) evidências baseadas em opiniões de especialistas ${ }^{(14)}$.

Por fim, foi conduzida a etapa de interpretação dos resultados, com categorização temática por similaridade de temas para facilitar a síntese e apresentação dos resultados da revisão.

\section{RESULTADOS}

Foram identificadas 346 referências distribuídas nas três bases de dados e foram excluídas 53 duplicatas. Em seguida, foi realizada a leitura dos títulos e resumos de 293 artigos e, desses, 37 eram elegíveis para leitura do texto na íntegra. Após leitura minuciosa, e aplicação dos critérios de exclusão estabelecidos, foram selecionados 15 artigos para a síntese das evidências, de acordo com o modelo PRISMA (Figura 1).

Figura 1 - Fluxograma dos artigos selecionados para compor a revisão integrativa.

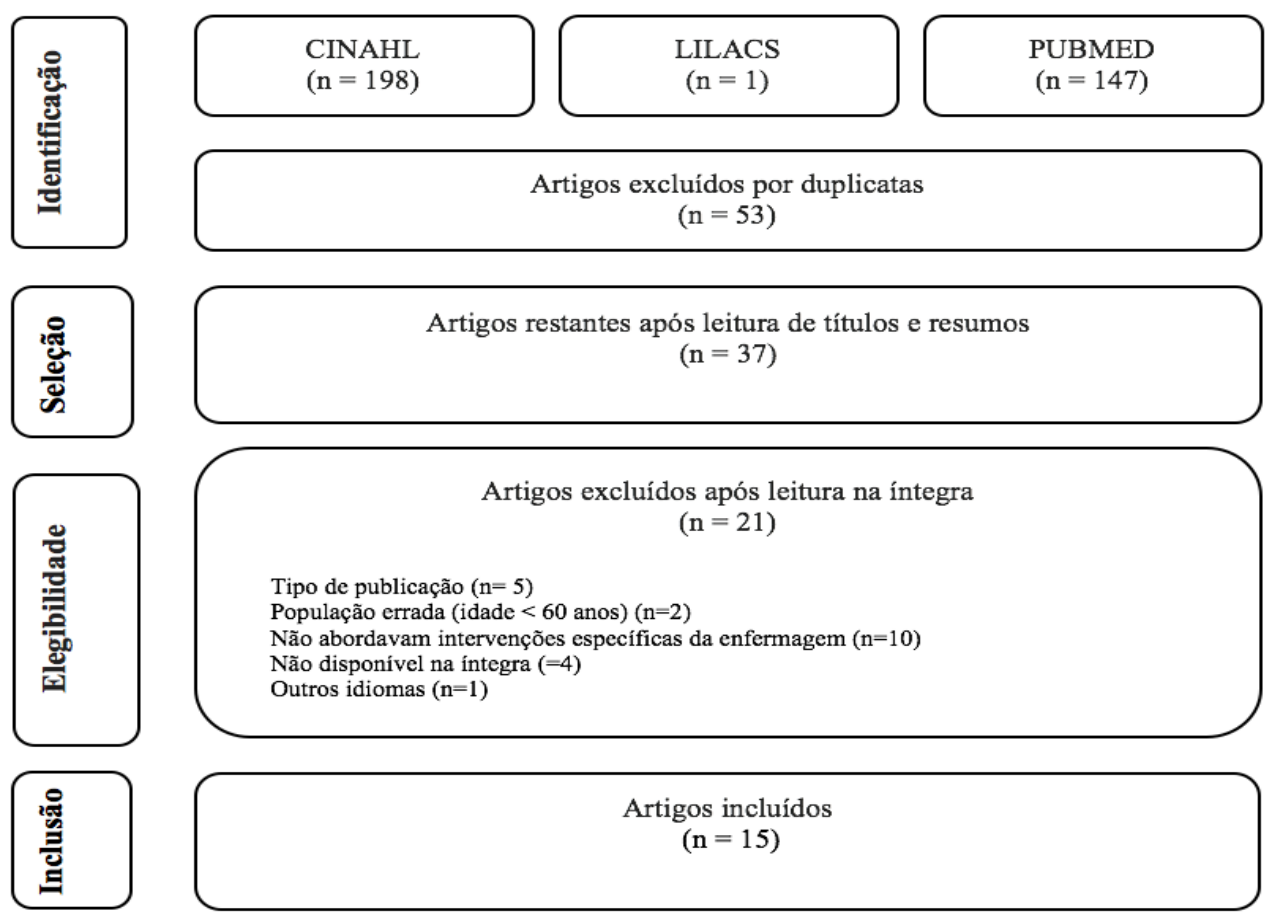

Fonte: Dados da pesquisa, 2020. 
Os 15 estudos incluídos foram realizados de 1999 a 2017, na Estados Unidos $(\mathrm{n}=3)$, Brasil $(\mathrm{n}=2)$, Nova Zelândia $(\mathrm{n}=2)$, Austrália $(\mathrm{n}=1)$, Bélgica $(\mathrm{n}=1)$, China $(\mathrm{n}=1)$, Espanha $(\mathrm{n}=1)$, Portugal $(\mathrm{n}=1)$, Reino Unido $(n=1)$, Suécia $(n=1)$ e Tailândia $(n=1)$. Dois estudos foram publicados em português $(n=2)$, um estudo em espanhol $(n=1)$ e os demais foram publicados em inglês $(n=12)$. A caracterização dos artigos foi apresentada no Quadro 2.

Quadro 2 - Caracterização dos artigos contemplando intervenções de enfermagem para prevenção de quedas em idosos na atenção primária, segundo: autores, ano de publicação, país, natureza/tipo do estudo, nível de evidência e participantes da pesquisa. Brasília, DF, Brasil, 2020.

\begin{tabular}{|c|c|c|c|c|c|}
\hline Autores & $\begin{array}{c}\text { Ano de } \\
\text { publicação }\end{array}$ & País & $\begin{array}{l}\text { Natureza e } \\
\text { tipo de } \\
\text { estudo }\end{array}$ & $\begin{array}{l}\text { Nível de } \\
\text { evidência }\end{array}$ & $\begin{array}{c}\text { Participantes } \\
\text { da pesquisa }\end{array}$ \\
\hline Allen A; Simpson JM ${ }^{15}$ & 1999 & $\begin{array}{l}\text { Reino } \\
\text { Unido }\end{array}$ & $\begin{array}{l}\text { Quantitativo, } \\
\text { experimental }\end{array}$ & 2 & Idosos \\
\hline Fortinsky RH et al ${ }^{16}$ & 2004 & $\begin{array}{l}\text { Estados } \\
\text { Unidos }\end{array}$ & $\begin{array}{l}\text { Quantitativo, } \\
\text { observacional }\end{array}$ & 4 & $\begin{array}{c}\text { Profissionais, } \\
\text { incluindo } \\
\text { enfermeiros }\end{array}$ \\
\hline Elley CR et al ${ }^{17}$ & 2007 & $\begin{array}{c}\text { Nova } \\
\text { Zelândia }\end{array}$ & $\begin{array}{l}\text { Quantitativo, } \\
\text { experimental }\end{array}$ & 2 & Idosos \\
\hline $\begin{array}{l}\text { Prat-González I; Fernández- } \\
\text { Escofet E; Martínez-Bustos } \\
\qquad S^{18}\end{array}$ & 2007 & Espanha & $\begin{array}{l}\text { Quantitativo, } \\
\text { observacional }\end{array}$ & 4 & Idosos \\
\hline Elley CR et al ${ }^{19}$ & 2008 & $\begin{array}{c}\text { Nova } \\
\text { Zelândia }\end{array}$ & $\begin{array}{l}\text { Quantitativo, } \\
\text { experimental }\end{array}$ & 2 & Idosos \\
\hline $\begin{array}{c}\text { Milisen K; Geeraerts A; } \\
\text { Dejaeger E }{ }^{20}\end{array}$ & 2009 & Bélgica & $\begin{array}{l}\text { Quantitativo, } \\
\text { observacional }\end{array}$ & 4 & $\begin{array}{c}\text { Idosos e } \\
\text { profissionais, } \\
\text { incluindo } \\
\text { enfermeiros }\end{array}$ \\
\hline Woo $\mathrm{J}$ et $\mathrm{al}^{21}$ & 2009 & China & $\begin{array}{l}\text { Quantitativo, } \\
\text { observacional }\end{array}$ & 4 & Idosos \\
\hline Macêdo ARC et al ${ }^{22}$ & 2012 & Brasil & $\begin{array}{l}\text { Quantitativo, } \\
\text { observacional }\end{array}$ & 4 & Idosos \\
\hline
\end{tabular}




\begin{tabular}{|c|c|c|c|c|c|}
\hline Resnick B et al ${ }^{23}$ & 2012 & $\begin{array}{l}\text { Estados } \\
\text { Unidos }\end{array}$ & $\begin{array}{l}\text { Quantitativo, } \\
\text { experimental }\end{array}$ & 2 & Idosos \\
\hline Cabrita MFG; José HMG ${ }^{24}$ & 2013 & Portugal & $\begin{array}{l}\text { Quantitativo, } \\
\text { observacional }\end{array}$ & 4 & Idosos \\
\hline $\begin{array}{c}\text { Romi H; } \\
\text { Wendy M; Terry } \mathrm{PH}^{25}\end{array}$ & 2014 & Austrália & $\begin{array}{l}\text { Quantitativo, } \\
\text { observacional }\end{array}$ & 4 & $\begin{array}{c}\text { Idosos e } \\
\text { profissionais, } \\
\text { incluindo } \\
\text { enfermeiros }\end{array}$ \\
\hline Fernandes $\mathrm{BKC}$ et $\mathrm{al}^{26}$ & 2016 & Brasil & $\begin{array}{l}\text { Quantitativo, } \\
\text { observacional }\end{array}$ & 4 & Idosos \\
\hline Boorgird C et al ${ }^{27}$ & 2017 & Tailândia & $\begin{array}{l}\text { Quantitativo, } \\
\text { experimental }\end{array}$ & 2 & Idosos \\
\hline Fahlström $\mathrm{G}$ et $\mathrm{al}^{28}$ & 2017 & Suécia & $\begin{array}{l}\text { Quantitativo, } \\
\text { experimental }\end{array}$ & 2 & Idosos \\
\hline $\begin{array}{c}\text { Reinoso H; McCaffrey RG; } \\
\text { Taylor DWM }{ }^{29}\end{array}$ & 2017 & $\begin{array}{l}\text { Estados } \\
\text { Unidos }\end{array}$ & $\begin{array}{l}\text { Quantitativo, } \\
\text { experimental }\end{array}$ & 2 & Idosos \\
\hline
\end{tabular}

Fonte: Dados da pesquisa, 2020.

Quanto às características metodológicas, a amostra foi composta por estudos de natureza quantitativa $(n=14)$, qualitativa $(n=1)$, do tipo experimental $(n=7)$ e observacional $(n=8)$, com nível de evidência $2(n=7)$ e $4(n=8)$, cujos participantes são idosos, enfermeiros e outros profissionais de saúde que atuam no contexto da atenção primária à saúde.
A análise dos artigos permitiu identificar duas temáticas recorrentes: i) intervenções de enfermagem relacionadas à avaliação e gestão do risco de quedas em idosos na atenção primária; ii) intervenções de enfermagem em programas multiprofissionais para prevenção de quedas em idosos na atenção primária (Quadro 3).

Quadro 3 - Intervenções de enfermagem para prevenção de quedas em idosos na atenção primária. Brasília, DF, Brasil, 2020.

Intervenções de enfermagem relacionadas à avaliação e gestão do risco de quedas em idosos na atenção primária

\section{Autores}

Principais resultados

https://doi.org/10.31011/reaid-2021-v.95-n.34-art.1104 Rev Enferm Atual In Derme v. 95, n. 34, 2021 e-021089 


\begin{tabular}{|c|c|}
\hline $\begin{array}{l}\text { Allen A; Simpson } \\
\mathrm{JM}^{(15)}\end{array}$ & $\begin{array}{l}\text { As enfermeiras conduziam um programa de prevenção de quedas para idosos } \\
\text { que caíram recentemente. O programa incluía: avaliação abrangente, visitas } \\
\text { domiciliares, vigilância dos fatores de risco ambientais, revisão de } \\
\text { medicamentos, encaminhamentos e exercícios de resistência. Outros } \\
\text { profissionais contribuíram para a construção dos componentes de avaliação } \\
\text { física, exercícios e revisão de medicamentos. }\end{array}$ \\
\hline et $\mathrm{al}^{(21)}$ & $\begin{array}{l}\text { Os enfermeiros desenvolveram uma ferramenta preditora de quedas em } \\
\text { idosos da comunidade. Realizaram identificação de risco pela triagem rápida } \\
\text { e encaminhamentos para avaliações detalhada. }\end{array}$ \\
\hline $\begin{array}{l}\text { Macêdo ARC et } \\
\mathrm{al}^{(22)}\end{array}$ & $\begin{array}{l}\text { Os enfermeiros desenvolveram ações relacionadas à identificação dos } \\
\text { aspectos da autonomia e da independência do idoso, identificação dos fatores } \\
\text { intrínsecos e extrínsecos relacionados a ocorrência de quedas. }\end{array}$ \\
\hline Resnick B et al $^{(23)}$ & $\begin{array}{l}\text { Os enfermeiros realizavam uma intervenção multicomponente que envolveu } \\
\text { a implementação de uma filosofia de cuidado na qual ajudavam os idosos em } \\
\text { tarefas funcionais e atividade física, avaliações de meio ambiente, definem } \\
\text { metas individualizadas, mentoria e motivação. }\end{array}$ \\
\hline $\begin{array}{l}\text { Cabrita MFG; José } \\
\mathrm{HMG}^{(24)}\end{array}$ & $\begin{array}{l}\text { Os enfermeiros realizaram visitas domiciliares, observaram a alta incidência } \\
\text { de quedas em idosos, identificaram os fatores que condicionam a saúde, } \\
\text { autonomia, como a patologia crônica múltipla, polimedicação, acidentes } \\
\text { domésticos, internações, alterações da estrutura familiar, avaliaram seu } \\
\text { ambiente físico, psíquico, social e ambiental. Forneceram orientações sobre a } \\
\text { segurança e autonomia e, se necessário, realizam encaminhamentos para } \\
\text { outros profissionais da equipe. }\end{array}$ \\
\hline $\begin{array}{l}\text { Fernandes BKC et } \\
\mathrm{al}^{(26)}\end{array}$ & $\begin{array}{l}\text { A identificação dos diagnósticos de enfermagem contribuiu para o início do } \\
\text { estabelecimento de ações de cuidados direcionadas aos idosos no intuito } \\
\text { minimizar a prevalência dos incidentes de queda. O enfermeiro intervém } \\
\text { através da prevenção estimulando ou monitorando a prática de exercícios } \\
\text { físicos, avaliando possíveis riscos domésticos (tapetes e pisos escorregadios) } \\
\text { e acompanhando as medicações que deixam o idoso mais vulnerável a } \\
\text { quedas. }\end{array}$ \\
\hline \multicolumn{2}{|c|}{$\begin{array}{l}\text { Intervenções de enfermagem em programas multiprofissionais para prevenção de quedas em } \\
\text { idosos na atenção primária }\end{array}$} \\
\hline
\end{tabular}




\begin{tabular}{|c|c|}
\hline Autores & Principais resultados \\
\hline $\begin{array}{lll}\text { Fortinsky } & \text { RH } & \text { et } \\
\mathrm{al}^{(16)} & & \end{array}$ & $\begin{array}{l}\text { A enfermagem identificou as barreiras ao abordar os sete fatores de risco } \\
\text { para quedas: dificuldades de marcha e transferência, distúrbios de equilíbrio, } \\
\text { uso de vários medicamentos, hipotensão postural, déficits sensoriais e } \\
\text { perceptivos, problemas com calçados e pés e riscos ambientais. Os } \\
\text { enfermeiros proporam estratégias de gestão de risco de queda, forneceram } \\
\text { educação aos prestadores de cuidados de saúde, intervenção direta e } \\
\text { encaminhamentos. }\end{array}$ \\
\hline Elley CR et al ${ }^{(17)}$ & $\begin{array}{l}\text { Os enfermeiros conduziram a avaliação e gestão dos riscos no ambiente } \\
\text { domiciliar para reduzir o risco de novas quedas, forneciam aconselhamento e } \\
\text { encaminhamentos (ex.: para o programa de exercícios conduzido por } \\
\text { educador físico ou fisioterapeuta). A intervenção incluia: i) avaliação de } \\
\text { saúde; ii) avaliação dos riscos domésticos; iii) avaliação da saúde óssea; iv) } \\
\text { encaminhamento para o The Otago Exercise Programme. }\end{array}$ \\
\hline $\begin{array}{l}\text { Prat-González I; } \\
\text { Fernández-Escofet } \\
\text { E; Martínez-Bustos } \\
S^{(18)}\end{array}$ & $\begin{array}{l}\text { A enfermagem atuava, junto com médicos, na implementação de um } \\
\text { protocolo para triagem dos fatores de risco para quedas. A intervenção } \\
\text { contemplou: exercícios e treinamentos para aumentar a força muscular e de } \\
\text { equilíbrio, revisão de medicamentos, correção da visão e de deficiência } \\
\text { auditiva, modificações no ambiente e recomendação de dieta adequada. }\end{array}$ \\
\hline Elley CR et al ${ }^{(19)}$ & $\begin{array}{l}\text { O enfermeiro com experiência em gerontologia visitava os idosos em seus } \\
\text { domicílios e realizavam avaliação de saúde padronizada, e utilizavam um } \\
\text { algoritmo para avaliação do risco de quedas. Também referenciava os idosos } \\
\text { para o The Otago Exercise Programme, sob responsabilidade de um } \\
\text { educador físico ou fisioterapeuta. Os enfermeiros observavam necessidades } \\
\text { de alterações ambientais. }\end{array}$ \\
\hline $\begin{array}{l}\text { Milisen } \\
\text { Geeraerts } \\
\text { Dejaeger } \mathrm{E}^{(20)}\end{array}$ & $\begin{array}{l}\text { O enfermeiro identificou os fatores de risco, forneceu a educação sobre a } \\
\text { importância do exercício/programa de exercício individualizado, realizou } \\
\text { encaminhamento pertinente de acordo com os fatores de risco, implementou } \\
\text { a educação sobre os efeitos de medicamentos em quedas, aconselhou a } \\
\text { prevenir a hipotensão postural e a usar calçados adequados, realizou o } \\
\text { checklist para segurança domiciliar e avaliação do ambiente. }\end{array}$ \\
\hline Romi H; & A introdução de um programa de intervenções múltiplas, com a atuação da \\
\hline
\end{tabular}




\begin{tabular}{|c|c|}
\hline $\begin{array}{l}\text { Wendy } \quad \mathrm{M} ; \quad \text { Terry } \\
\mathrm{PH}^{(25)}\end{array}$ & $\begin{array}{l}\text { enfermagem, com enfoque de fornecer educação culturalmente específica } \\
\text { sobre o risco de quedas, mudanças comportamentais eficazes para a } \\
\text { prevenção de quedas e suas consequências. }\end{array}$ \\
\hline 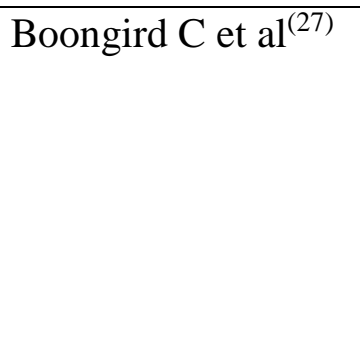 & $\begin{array}{l}\text { Os enfermeiros integravam um programa multiprofissional de exercícios } \\
\text { domiciliares que contemplava intervenções múltiplas. Eles realizavam visitas } \\
\text { domiciliares, obtinham informações sobre os fatores de risco ambientais, } \\
\text { incentivavam a prática dos exercícios físicos e a adoção de comportamentos } \\
\text { seguros nessas atividades. }\end{array}$ \\
\hline $\mathrm{m} \mathrm{G}$ et $\mathrm{al}^{(2}$ & $\begin{array}{l}\text { Os auxiliares de enfermagem atuavam em conjunto com fisioterapeutas em } \\
\text { programas de exercícios domiciliares. Os auxiliares de enfermagem } \\
\text { supervisionavam o desempenho das atividades durante oito visitas } \\
\text { domiciliares, ajudavam e encorajavam a performance das atividades. }\end{array}$ \\
\hline $\begin{array}{l}\text { Reinoso H; } \\
\text { McCaffrey RG; } \\
\text { Taylor DWM }\end{array}$ & $\begin{array}{l}\text { Enfermeiros participaram do desenvolvimento e implementação de um } \\
\text { programa de prevenção de risco de queda. Suas ações consistiam em } \\
\text { identificar os fatores de risco durante a triagem, gerenciar e monitorar a } \\
\text { hipotensão, otimizar a segurança do ambiente e modificações dos calçados. } \\
\text { Juntamente com outros profissionais, realizaram a revisão minuciosa e ajuste } \\
\text { de doenças e tratamentos farmacológicos. }\end{array}$ \\
\hline
\end{tabular}

Fonte: Dados da pesquisa, 2020.

\section{DISCUSSÃO}

O profissional enfermeiro tem contribuição importante no cuidado a saúde do idoso na atenção primária. Entre as suas atribuições, destacam-se realizar atenção integral aos idosos, desempenhar assistência no ambiente domiciliar e realizar avaliação multidimensional do idoso. A sua atuação deve contemplar os principais problemas que podem comprometer a saúde dessa população, por exemplo, as quedas ${ }^{(30)}$.
Neste sentido, esta revisão integrativa buscou identificar as intervenções de enfermagem para prevenção de quedas em idosos na atenção primária. Os artigos incluídos foram publicados entre 1999 e 2017, realizados em diversos países. As características heterogêneas em relação à data de publicação e país, permitem inferir que os enfermeiros que atuam na atenção primária têm direcionado o olhar para a as quedas em idosos e aprimorado sua atuação ao longo dos anos para prevenir estes eventos no público em questão. 
A análise dos estudos que integraram a amostra desta revisão permitiu identificar duas temáticas recorrentes nos artigos, a primeira sobre as intervenções de enfermagem relacionadas à avaliação e gestão do risco de quedas, e a segunda abrange as intervenções de enfermagem no contexto de programas multiprofissionais para prevenção de quedas em idosos na atenção primária.

Um estudo realizado nos Estados Unidos apontou a atuação da enfermagem na identificação dos fatores de risco para quedas, contemplando as dificuldades de marcha e transferência, distúrbios de equilíbrio, uso de vários medicamentos, hipotensão postural, déficits sensoriais e perceptivos, problemas com calçados e pés e riscos ambientais. Após identificar os fatores de risco, os enfermeiros estabeleciam estratégias de gestão do risco de queda, com intervenções diretas, encaminhamentos pertinentes e oferta de capacitação para a equipe de saúde ${ }^{(16)}$.

Outro artigo de pesquisa realizada na Bélgica demonstrou que os enfermeiros atuavam na identificação dos fatores de risco, oferta de orientações sobre a importância dos exercícios físicos, efeitos dos medicamentos que podem desencadear quedas, prevenção da hipotensão postural e uso de calçados adequados. Além disso, também realizavam avaliação ambiental e aplicavam um checklist para segurança domiciliar ${ }^{(20)}$.
De fato, os enfermeiros exercem papel relevante na identificação do risco de quedas em idosos. Um estudo de revisão integrativa, reiterou a importância dos enfermeiros, que mantém maior vínculo com os idosos no seu ambiente domiciliar, na identificação dos fatores de riscos ambientais e aqueles predisponentes para quedas e orientações que contemplem as necessidades reais do idoso, incentivo a prática de exercícios físicos para manutenção do equilíbrio e promoção de um ambiente domiciliar seguro que mantenha a autonomia e independência dos idosos ${ }^{(31)}$.

Também foi identificada a atuação de enfermeiras na implementação e condução de programas de prevenção de quedas em idosos. No Reino Unido, o programa contemplava visitas domiciliares, avaliação integral do idoso, identificação dos fatores de risco com ênfase nos fatores ambientais e revisão de medicamentos ${ }^{(15)}$. Nos Estados Unidos, as enfermeiras atuavam na otimização da segurança ambiental, modificação de calçados e revisão de medicamentos ${ }^{(29)}$.

Um aspecto que chamou a atenção nos estudos mencionados foi a possibilidade de enfermeiros atuarem inclusive no ambiente domiciliar dos idosos. A visita domiciliar é considerada uma atividade externa à unidade básica de saúde, é um meio de cuidado que dispõe de tecnologia leve e leve-dura, que envolvem um cuidado mais humanizado, acolhedor que requer sensibilidade do 
profissional para identificar as necessidades $^{(32)}$.

Segundo o Ministério da Saúde ${ }^{(33)}$, é evidente a necessidade do enfermeiro dispor do cuidado integral e humanizado à pessoa idosa no contexto do seu domicílio para prestar uma assistência individualizada para cada idoso, promover ações de identificação dos fatores de riscos, a ocorrência de quedas durante a triagem, otimizar a segurança do ambiente, orientar a pessoa idosa e seus familiares quanto aos riscos de queda, com linguagem clara para melhor compreensão; modificar os calçados e fornecer educação sobre a importância do exercício.

Os artigos revelaram que nas visitas domiciliares, além da avaliação dos fatores intrínsecos e extrínsecos para quedas, e identificação dos riscos ambientais, os enfermeiros ofereciam orientações sobre a segurança ambiental, promoção da autonomia dos idosos e avaliavam a necessidade de encaminhá-los para avaliação de outros profissionais de saúde ${ }^{(22-24)}$. Para auxiliar na avaliação do risco de quedas, os enfermeiros também aplicam avaliações de saúde padronizadas e algoritmos baseados em evidências para avaliar o risco de quedas ${ }^{(19)}$.

É importante ressaltar a necessidade de enfermeiros utilizarem ferramentas que favoreçam a identificação do risco de quedas em idosos na sua prática clínica, como a determinação de diagnósticos de enfermagem $^{(26)}$. Neste sentido, uma pesquisa realizada no Distrito Federal - Brasil, com objetivo de avaliar o diagnóstico de enfermagem de Risco de Quedas em idosos na atenção primária, evidenciou a importância do enfermeiro utilizar esse recurso em conjunto com outros instrumentos para avaliação do risco de quedas. A determinação do diagnóstico Risco de Quedas possibilita a avaliação inicial do risco e favorece a tomada de decisão do enfermeiro na escolha das intervenções necessárias ${ }^{(34)}$.

Outro aspecto que chamou atenção nos artigos foi a tomada de decisão do enfermeiro sobre encaminhar o idoso para avaliação de outros profissionais de saúde. Apesar das intervenções de enfermagem serem essenciais para a prevenção das quedas, deve-se assumir que estes eventos são muito complexos, de origem multifatorial, e que só é possível alcançar bons resultados com o idoso se houver a avaliação e a intervenção multiprofissional.

Nesse sentido, a segunda temática que emergiu da análise dos resultados foi sobre as intervenções de enfermagem no contexto de programas multiprofissional para prevenção de quedas. Mesmo que as intervenções de enfermagem identificadas sejam similares às apresentadas nos demais artigos, é interessante verificar como essas intervenções podem contribuir para o alcance de objetivos no contexto do trabalho multiprofissional. 
Em um estudo realizado na Espanha, os enfermeiros atuavam em conjunto com médicos na implementação de um protocolo para triagem do risco de quedas, revisão de medicamentos, correção da visão e de deficiências auditivas e modificações no ambiente $^{(18)}$. Já na Austrália, os enfermeiros contribuíam na educação sobre o risco de quedas, mudanças comportamentais e estabelecimento de metas para aumentar a motivação e a autoeficácia dos idosos ${ }^{(25)}$.

Em outro estudo, desenvolvido na Tailândia, os enfermeiros integravam um programa de exercícios domiciliares, no qual eles realizavam visitas domiciliares para identificação de fatores de risco ambientais, incentivavam a prática dos exercícios físicos e a adoção de comportamentos seguros ${ }^{(27)}$.

A revisão também contemplou o The Otago Exercise Programme, no qual enfermeiros com experiência em gerontologia conduziam a avaliação e a gestão dos riscos ambientais no domicílio, realizavam aconselhamentos e encaminhavam os idosos para o programa de exercícios físicos conduzido por fisioterapeutas e educadores físicos, com finalidade de melhorar o equilíbrio, a força muscular e a capacidade para caminhar ${ }^{(19)}$. Ainda no contexto do programa, auxiliares de enfermagem contribuíam na supervisão dos idosos durante a execução dos exercícios ${ }^{(17)}$.
A participação dos enfermeiros em programas cujo componente central são os exercícios físicos merece atenção, uma vez que tem sido evidenciado que a prática regular de atividade física como intervenção isolada está associada a uma melhor condição de saúde dos idosos e uma menor incidência de quedas $^{(35)}$. Mesmo que não seja competência dos enfermeiros atuarem na prescrição e orientação para prática desses exercícios, verificou-se que eles podem contribuir em outros aspectos desses programas, como na avaliação integral dos idosos, na identificação e gestão do risco de quedas (especialmente o risco ambiental), além de oferecer orientações para os idosos e cuidadores.

Aponta-se como possível limitação deste estudo a indisponibilidade de alguns artigos para leitura na íntegra, porém, não inviabilizou o alcance do objetivo proposto. Ademais, esta revisão contribuiu para preencher uma lacuna existente até o momento quanto à síntese de estudos que abordam as intervenções de enfermagem pertinentes para prevenção das quedas em idosos atendidos na atenção primária.

\section{CONSIDERAÇÕES FINAIS}

A revisão integrativa possibilitou realizar uma síntese de estudos sobre as intervenções de enfermagem para prevenção 
de quedas em idosos na atenção primária. Estas intervenções consistem em: avaliar/identificar fatores de risco intrínsecos e extrínsecos para quedas, com ênfase nos fatores modificáveis; identificar e gerir riscos ambientais, na oportunidade das visitas domiciliares; oferecer orientações para os idosos/cuidadores quanto a promoção da segurança no ambiente domiciliar e necessidade de mudanças comportamentais; promover educação permanente para a equipe de saúde sobre prevenção de quedas; conduzir e/ou participar de programas multiprofissionais com a finalidade de prevenir as quedas.

Acredita-se que estes achados podem contribuir para o planejamento das ações dos profissionais de enfermagem que se deparam cotidianamente com o desafio de prevenir as quedas na população idosa no contexto domiciliar e das unidades de saúde.

\section{REFERÊNCIAS}

1. World Health Organization. WHO global report on falls prevention in older age. Geneva, Switzerland: World Health Organization; 2010. 47 p.

2. World Health Organization. Falls [Internet]. 2020 [citado 2020 ago 12]. Disponível em: https://www.who.int/news-room/factsheets/detail/falls

3. Soares WJS, Moraes SA, Ferriolli E, Perracini MR, Soares WJS, Moraes SA et al. Fatores associados a quedas e quedas recorrentes em idosos: estudo de base populacional. Rev. bras. geriatr. gerontol. [Internet]. 2014 [citado 2020 set 22]; 17(1): 49-60. DOI:
https://doi.org/10.1590/S180998232014000100006

4. Smith AA, Silva AO, Rodrigues RAP, Moreira MASP, Nogueira JA, Tura LFR. Assessment of risk of falls in elderly living at home. Rev. LatinoAm. $\quad$ Enfermagem [Internet]. 2017 [citado 2020 ago 25]; 25: e2754. DOI: https://doi.org/10.1590/1518-8345.0671.2754

5. Pimenta CJL, Lima RJ, Costa TF, Bezerra TA, Martins KP, Leal NPR et al. Prevalência de quedas em idosos atendidos em um centro de atenção integral. Reme, Rev. Min. Enferm [Internet]. 2017 [citado 2020 ago 25]; 21(0):1-7. DOI: http://www.dx.doi.org/10.5935/14152762.20170055

6. Garcia TR, organizador. Classificação internacional para a Prática de Enfermagem CIPE: versão 2019/2020. Porto Alegre: ArtMed; 2020.

7. NANDA International. NANDA International nursing diagnoses: definitions and classification 2018-2020. Herdman TH, Kamitsuru S, organizadores. Artmed. Porto Alegre: Artmed; 2018.

8. Luzia MF, Almeida MA, Lucena AF, Luzia MF, Almeida MA, Lucena AF. Nursing care mapping for patients at risk of falls in the Nursing Interventions Classification. Rev. esc. enferm. USP [Internet]. 2014 [citado 2020 sept 22]; 48(4): 632-640. DOI: https://doi.org/10.1590/S0080623420140000400009

9. Brasil. Ministério da Saúde. Protocolo de Prevenção de Quedas [Internet]. [citado em 2020 ago 11]. Disponível em: https://www20.anvisa.gov.br/segurancadopaciente /index.php/publicacoes/item/prevencao-de-quedas

10. Fernandes BKC, Clares JWB, Borges CL, Nóbrega MML, Freitas MC, Fernandes BKC et al. Nursing diagnoses for institutionalized elderly people based on Henderson's theory. Rev. esc. enferm. USP [Internet]. 2019 [citado 2020 ago 12]; 53: e03472. DOI: https://doi.org/10.1590/s1980-

220x2018004103472

11. Luzia MF, Victor MAG, Lucena AF. Nursing Diagnosis Risk for falls: prevalence and clinical profile of hospitalized patients. Rev. Latino-Am. 
Enfermagem [Internet]. 2014 [citado 2020 set 22]; 22(2): 262-268.

DOI:

https://doi.org/10.1590/0104-1169.3250.2411

12. Mendes KDS, Silveira RCCP, Galvão CM. Revisão integrativa: método de pesquisa para a incorporação de evidências na saúde e na enfermagem. Texto contexto - enferm. [Internet]. 2008 [citado 2020 abr 15]; 17(4):758-764. DOI: https://doi.org/10.1590/S0104-

07072008000400018

13. Peters MDJ, Godfrey C, McInerney P, Munn Z, Tricco AC, Khalil H. Chapter 11: Scoping Reviews (2020 version). In: Aromataris E, Munn Z (Ed.). JBI Manual for Evidence Synthesis, JBI, 2020. Disponível

https://synthesismanual.jbi.global.

14. Souza MT, Silva MD, Carvalho R. Integrative review: what is it? How to do it? Einstein (São Paulo) [Internet]. 2010 [citado 2020 abr 15]; 8(1): 102-106. DOI: https://doi.org/10.1590/s167945082010rw1134

15. Allen A, Simpson J. A primary care based fall prevention programme. Physiother. Theory Pract [Internet]. 2009 [citado 2020 abr 29]; 10(15): 121-33.

DOI:

https://doi.org/10.1080/095939899307810

16. Fortinsky RH, Iannuzzi-Sucich M, Baker DI, Gottschalk M, King MB, Brown CJ, Tinetti ME. Fall-risk assessment and management in clinical practice: views from healthcare providers. J Am Geriatr Soc. [Internet]. 2004 [citado 2020 abr 29]; 52(9):1522-6.

DOI: https://doi.org/10.1111/j.1532-5415.2004.52416.x

17. Elley CR, Robertson MC, Kerse NM, Garrett S, McKinlay E, Lawton B et al. Falls Assessment Clinical Trial (FACT): design, interventions, recruitment strategies and participant characteristics. BMC Public Health [Internet]. 2007 [citado 2020 abr 29]; 7:185. DOI: https://doi.org/10.1186/1471-2458-7-185

18. Prat-González I, Fernández-Escofet E, Martínez-Bustos S. Assessment of risk of falls in the elderly through a screening protocol in primary care. Enferm Clin. [Internet]. 2007 [citado 2020 apr 29]; 17(3):128-33. DOI: https://doi.org/10.1016/S1130-8621(07)71783-7
19. Elley CR, Robertson MC, Garrett S, Kerse NM, McKinlay E, Lawton B et al. Effectiveness of a falls-and-fracture nurse coordinator to reduce falls: a randomized, controlled trial of at-risk older adults. J Am Geriatr Soc [Internet]. 2008 [citado 2020 abr 29]; 56(8):1383-9. DOI: https://doi.org/10.1111/j.1532-5415.2008.01802.x

20. Milisen K, Geeraerts A, Dejaeger E. Use of a fall prevention practice guideline for communitydwelling older persons at risk for falling: A feasibility study. Gerontology [Internet]. 2009 [citado 2020 apr 29]; 55(2):169-78. DOI: https://doi.org/10.1159/000165172

21. Woo J, Leung J, Wong S, Kwok T, Lee J, Lynn H. Development of a simple scoring tool in the primary care setting for prediction of recurrent falls in men and women aged 65 years and over living in the community. J Clin Nurs [Internet]. 2009 [citado 2020 may 01]; 18(7):1038-48. DOI: https://doi.org/10.1111/j.1365-2702.2008.02591.x

22. Macedo ARC, Alves DS, Silva Junior FJG, Rocha FCV, Carvalho PMG. Evaluation of daily life activities in primary care after falls, in elderly persons. J Nurs UFPE / Rev Enferm UFPE [Internet]. 2012 [citado 2020 may 01]; 6(3):61926. DOI: https://doi.org/10.5205/reuol.216316218-1-LE.0603201217

23.Resnick B, Galik E, Gruber-Baldini AL, Zimmerman S. Falls and fall-related injuries associated with function-focused care. Clin Nurs Res. [Internet]. 2012 [citado 2020 may 01]; 21(1):43-63.

DOI:

https://doi.org/10.1177/1054773811420060

24. Cabrita MFG, José HMG. O idoso na equipe de cuidados continuados integrados: programa de enfermagem para prevenção de quedas. Rev Enferm UFPE On Line [Internet]. 2013 [citado 2020 maio 01]; 7(1):96-103. DOI: https://doi.org/10.5205/1981-8963-

v7i1a10209p96-103-2013

25. Haas R, Mason W, Haines TP. Difficulties Experienced in Setting and Achieving Goals by Participants of a Falls Prevention Programme: A Mixed-Methods Evaluation. Physiother Can. [Internet]. 2014 [citado 2020 may 01]; 66(4):41322. DOI: https://doi.org/10.3138/ptc.2013-30BC 
26. Fernandes BKC, Freitas MC, Galiza FT, Borges CL, Queiroz SMB, Rocha VA. Diagnósticos de enfermagem para idosos em uso de medicamentos orais. Rev enferm UFPE On line [Internet]. 2016 [citado 2020 maio 02]; 10(4):1179-84. DOI: https://doi.org/10.5205/reuol.8464-74011-1SM.1004201602

27. Boongird C, Keesukphan P, Phiphadthakusolkul S, Rattanasiri S, Thakkinstian A. Effects of a simple home-based exercise program on fall prevention in older adults: A 12month primary care setting, randomized controlled trial. Geriatr Gerontol Int. [Internet]. 2017 [citado 2020 may 01]; 17(11):2157-63. DOI: https://doi.org/10.1111/ggi.13052

28. Fahlström G, Kamwendo K, Forsberg J, Bodin L. Fall prevention by nursing assistants among community-living elderly people. A randomised controlled trial. Scand J Caring Sci [Internet]. 2018 [citado 2020 may 01]; 32(2):575-85. DOI: https://doi.org/10.1111/scs.12481

29. Reinoso H, McCaffrey RG, Taylor DWM. Mitigating fall risk: A community fall reduction program. Geriatr Nurs. [Internet]. 2018 [citado 2020 may 01]; 39(2):199-203. DOI: https://doi.org/10.1016/j.gerinurse.2017.08.014

30. Brasil. Ministério da Saúde. Secretaria de Atenção à Saúde. Departamento de Atenção Básica. Envelhecimento e saúde da pessoa idosa. Brasília: Ministério da Saúde; 2007. 192 p.

31. Miranda DP, Santos TD, Espírito Santo FH, Pinho CL, Barreto EA. Quedas em idosos em ambiente domiciliar: uma revisão integrativa. REAID [Internet]. 2016 [citado 2020 maio 01];
120-9. DOI: https://doi.org/10.31011/reaid-2017v.2017-n.0-art.560

32. Nogueira IS, Previato GF, Scolari GA Souza, Gomes ACO, Carreira L, Baldissera VDA. Home intervention as a tool for nursing care: evaluation of the satisfaction of the elderly. Rev. Gaúcha Enferm. [Internet]. 2016 [citado 2020 oct 31]; 37: e68351. DOI: http://dx.doi.org/10.1590/19831447.2016.esp.68351

33. Brasil. Ministério da Saúde. Orientações técnicas para a implementação de linha de cuidado para atenção integral à saúde da pessoa idosa: no Sistema Único de Saúde - SUS. Brasília (DF): Ministério da Saúde; 2018. 95p.

34. Santos PHFS, Stival MM, Santos WS, Volpe CRG, Rehem TCMSB, Funghetto SS. Nursing diagnosis Risk for Falls in the elderly in primary healthcare. Rev. Bras. Enferm. [Internet]. 2020 [citado $2020 \mathrm{dez} 22$ ]; 73(Suppl 3): e20180826. DOI: http://dx.doi.org/10.1590/0034-7167-20180826

35. Bento JR, Sousa ND. Ejercicio físico en la prevención de caídas del anciano de la comunidad. Rev bras med fam comunidade [Internet]. 2017 [citado $2020 \mathrm{dez} 22$ ];12(39):1-11. DOI: http://dx.doi.org/10.5712/rbmfc12(39) 1658

Autor Correspondente: Paulo Henrique Fernandes dos Santos

Endereço: Universidade de Brasília, Faculdade Ciências da Saúde Asa Norte, CEP 70910900 - Brasília, DF, Brasil E-mail: paulofs@unb.br

Submissão: 2021-05-05

Aprovado: 2021-05-30 